\title{
Comparison of respiratory pathogen colonization and antimicrobial susceptibility in people with cystic fibrosis bronchiectasis versus non-cystic fibrosis bronchiectasis: a protocol for a systematic review
}

\author{
Salony Verma', Joseph L. Mathew ${ }^{2^{*}}$ (i) and Pallab Ray ${ }^{1}$
}

\begin{abstract}
Background: Both cystic fibrosis (CF) and non-cystic fibrosis bronchiectasis are characterized by permanent bronchial dilation, impaired mucociliary clearance, and development of chronic colonization and infection. Although the major airway microbiota in both CF and non-CF bronchiectasis may be similar, there are some differences in clinical and microbiologic features. There may also be differences in antibiotic susceptibility patterns between the CF and non-CF populations. Therefore, analysis and comparison of the microbiota and antibiotic susceptibility pattern in CF bronchiectasis versus non-CF bronchiectasis would help to improve the management of both conditions.

Methods: Two authors will independently search the electronic databases PubMed, EMBASE, the Cochrane Library, and LIVIVO, for studies reporting bacterial colonization of the respiratory tract in adults and children diagnosed with bronchiectasis in either CF or non-CF. We will include studies examining any respiratory tract specimen, using conventional bacterial culture or other specialized techniques such as molecular methods. We will also examine the antimicrobial susceptibility patterns in people with CF bronchiectasis versus non-CF bronchiectasis. The authors will independently assess the risk of bias in each included study using the Newcastle Ottawa Scale (NOS). We will present the data with descriptive statistics and provide pooled estimates of outcomes, wherever it is feasible to perform meta-analysis. Heterogeneity in studies will be explored by visual inspection of forest plots as well as using the Higgins and Thompson $P^{2}$ method. We will contact the corresponding authors of studies where data is/are missing and try to obtain the missing data. We will undertake sensitivity analysis to explore the impact of study quality and subgroup analysis based on pre-set criteria. We will prepare a summary of findings' table and assess the confidence in the evidence using the GRADE methodology.

(Continued on next page)
\end{abstract}

\footnotetext{
*Correspondence: joseph.I.mathew@gmail.com

${ }^{2}$ Department of Pediatrics, Post Graduate Institute of Medical Education and Research, Chandigarh 160012, India

Full list of author information is available at the end of the article
}

(c) The Author(s). 2021 Open Access This article is licensed under a Creative Commons Attribution 4.0 International License, which permits use, sharing, adaptation, distribution and reproduction in any medium or format, as long as you give appropriate credit to the original author(s) and the source, provide a link to the Creative Commons licence, and indicate if changes were made. The images or other third party material in this article are included in the article's Creative Commons licence, unless indicated otherwise in a credit line to the material. If material is not included in the article's Creative Commons licence and your intended use is not permitted by statutory regulation or exceeds the permitted use, you will need to obtain permission directly from the copyright holder. To view a copy of this licence, visit http://creativecommons.org/licenses/by/4.0/. The Creative Commons Public Domain Dedication waiver (http://creativecommons.org/publicdomain/zero/1.0/) applies to the data made available in this article, unless otherwise stated in a credit line to the data. 
(Continued from previous page)

Discussion: To date, there are no locally applicable evidence-based guidelines for antimicrobial treatment of nonCF bronchiectasis patients. In general, treatment is based on extrapolation of evidence in people with CF bronchiectasis. An insight into the microbiota and antimicrobial susceptibility patterns in the two conditions would facilitate appropriate rather than empiric antimicrobial therapy and hopefully reduce the burden of antimicrobial resistance created by rampant usage of antibiotics.

Systematic review registration: The protocol has been registered in PROSPERO on July 26, 2020 (PROSPERO registration number: CRD42020193859).

Keywords: Bronchiectasis, Cystic fibrosis bronchiectasis, Non-CF bronchiectasis, Antimicrobial susceptibility, Bacterial colonization, Antibiotic, Resistance, Susceptibility

\section{Background}

Cystic fibrosis (CF) is an autosomal recessive disorder caused by mutations in cystic fibrosis transmembrane conductance regulator (CFTR) gene. This gene regulates the activity of sodium and chloride channels across the epithelial cells, thereby facilitating appropriate hydration of mucins and effective muco-ciliary clearance in various organs [1]. Impaired secretion of chloride and bicarbonate ions due to CFTR mutations leads to the formation of thick mucus, which is difficult to clear [2]. This predisposes CF patients to pulmonary bacterial infections caused by Staphylococcus aureus, Pseudomonas aeruginosa, Haemophilus influenzae, or Burkholderia cepacia complex (Bcc) [3]. The inflammatory response to recurrent infections eventually leads to bronchiectasis, characterized by permanent bronchial dilation. This causes bacterial adherence, increased bacterial load, and the development of chronic infection. The bacteria gradually adapt to these conditions by forming biofilms, protecting them from phagocytosis as well as penetration of antibiotics [4].

Besides CF, bronchiectasis is associated with various other conditions such as immunodeficiency disorders, autoimmune diseases, ciliary abnormalities, connective tissue diseases, airway injury, malignancy, inflammatory bowel disease, alpha-1 antitrypsin deficiency, or hypersensitivity (allergic bronchopulmonary aspergillosis). These are collectively termed as non-CF bronchiectasis [5].

There are many similarities between $\mathrm{CF}$ and non-CF bronchiectasis. Both are associated with severe airway inflammation, mucus obstruction, reduced lung function, progression over time, and frequent pulmonary exacerbations $[6,7]$. However, there are also many differences between the two conditions.

Various biological specimens have been analyzed for identifying bacterial colonization patterns in people with bronchiectasis. Sputum is the preferred specimen for culturing bacteria and is also tested for acid-fast bacilli in non-CF bronchiectasis [5]. Bronchoalveolar lavage (BAL) is reserved for patients who are unable to produce sputum or when sputum does not yield organisms.
Epithelial lining fluid (ELF) is another respiratory specimen used for microbiologic analysis.

The major microbiota colonizing the airways are similar in both CF and non-CF bronchiectasis. Staphylococcus aureus, Pseudomonas aeruginosa, Burkholderia cepacia complex, Haemophilus influenzae, Stenotrophomonas maltophilia, and Achromobacter xylosoxidans are commonly associated with CF bronchiectasis [8] while Haemophilus influenzae, Pseudomonas aeruginosa, Moraxella catarrhalis, or non-tuburculous Mycobacteria (NTM) are the predominant bacterial species associated with non-CF bronchiectasis [5, 9]. Gram-positive bacteria including Streptococcus pneumoniae and Staphylococcus aureus are rarely associated with non-CF bronchiectasis unlike CF bronchiectasis [10]. Further, the core microbiota in both the conditions are similar in childhood but diverge by adulthood $[6,11]$.

Antibiotics are the mainstay of treatment of bronchiectasis in both CF and non-CF patients. The choice of antibiotics is based on an understanding of the predominant respiratory tract colonizers in individual patients, as well as local antimicrobial susceptibility testing (AST) patterns. The appropriate use of antibiotics is associated with improved pulmonary function and survival [12].

AST may predict the success or failure of specific antibiotics, by sorting out the resistant bacteria from the susceptible ones on the basis of Minimal Inhibitory Concentration (MIC) breakpoints. These are determined by breakpoint committees such as the European Committee on Antimicrobial Susceptibility Testing (EUCAST) or the Clinical and Laboratory Standards Institute (CLSI). However, the epidemiological cut-off is determined using the susceptibility data from the wildtype population and does not take into consideration any mutant strains [13], which are commonly encountered in the mucus-obstructed airways of CF patients. Therefore, people with CF bronchiectasis often receive antibiotics in higher doses and for longer duration, compared to non-CF bronchiectasis [14]. So clinicians cannot rely only on such data for prescribing empirical therapy to the CF patients. There are several other 
factors, responsible for differences in antibiotic susceptibility patterns between $\mathrm{CF}$ and non-CF populations. Bacteria in CF airways respond to the deficient oxygen or nutrient conditions by slowing down their growth rate or by altering their metabolism [4], which fosters resistance to several antibiotics. Therefore, antibiotics acting on the cell-wall may not be effective in eradicating bacteria that are not actively dividing or are growing slowly. Many bacteria form biofilms, which make them impervious to antibiotics [15]. In addition, different colonial types of bacteria such as small colony variants (SCV) are observed in the respiratory specimens of CF patients [16, 17], which are often missed in routine laboratory testing. A single specimen from CF patients may contain mixed populations of a single organism with different antibiotic susceptibility profile [18].

Therefore, a detailed comparison of respiratory pathogen colonization in people with CF bronchiectasis and non-CF bronchiectasis, and the antimicrobial susceptibility patterns in them, could improve management of both conditions.

\section{Aim and objectives of the systematic review}

This systematic review aims to compare the microbiota and antimicrobial susceptibility profile in people with $\mathrm{CF}$ bronchiectasis versus non-CF bronchiectasis. We propose to undertake a systematic review of literature to address the following research questions:

1. What are the bacteria colonizing the respiratory tract in people with cystic fibrosis bronchiectasis, compared to non-cystic fibrosis bronchiectasis?

2. How does the antibiotic susceptibility profile of specific bacteria, differ between people with CF bronchiectasis versus non-CF bronchiectasis?

\section{Methods/design}

The protocol of this systematic review has been registered in the PROSPERO database, the International prospective register of systematic reviews on July 26, 2020 (CRD42020193859). This protocol is in accordance with the Preferred Reporting Item for Systematic Review and Metaanalysis (PRISMA-P) guidelines (Additional file 1) [19].

\section{Types of studies}

The data required for this review could be available in observational studies including cohort studies or casecontrol studies (case arm), one or other arm of controlled clinical trials (randomized or non-randomized), or case series.

\section{Types of participants}

Adults and children diagnosed with CF bronchiectasis or non-CF bronchiectasis.

\section{Inclusion criteria}

We will include studies reporting bacterial colonization of the respiratory tract determined by examination of any respiratory tract specimen, by conventional bacterial culture or molecular techniques, and/or antimicrobial susceptibility testing by any method.

\section{Exclusion criteria}

We will exclude the following studies:

1. Those which include people with CF or non-CF conditions, but without bronchiectasis.

2. Those in which data of patients with and without bronchiectasis cannot be distinguished.

3. Those in which the underlying cause(s) of bronchiectasis cannot be distinguished as $\mathrm{CF}$ or non-CF.

4. Those wherein multiple clinical conditions were studied, but it is not possible to obtain or analyze data for CF or non-CF bronchiectasis.

5. Those reporting microbiology data wherein the underlying clinical condition(s) are not specified.

6. Those in which non-standard culture methods were used to identify organisms.

7. Case series with less than 10 participants.

8. Studies conducted in animals or animal models, or studies wherein already identified organisms were evaluated further for genotypic or phenotypic characteristics.

\section{Comparisons considered in this review}

1. Clinical or microbiological studies of any design (observational, controlled clinical trials, or case series), reporting bacterial colonization of the respiratory tract (from any type of biological specimen) in CF and non-CF bronchiectasis, and/or antimicrobial susceptibility in both types of patients. Such studies will be considered direct comparisons.

2. Clinical or microbiological studies of any design (observational, controlled clinical trials, or case series), reporting bacterial colonization of the respiratory tract, and/or antimicrobial susceptibility in either CF or non-CF patients, within a 3-year period. Such studies will be considered indirect comparisons.

3. Clinical or microbiological studies of any design (observational, controlled clinical trials, or case series), reporting bacterial colonization of the respiratory tract, and/or antimicrobial susceptibility in either CF or non-CF patients, within any time period, if they are from the same institution. Such studies will also be considered indirect comparisons. 


\section{Types of outcome measures}

1. Bacteria identified in the respiratory tract in people with $\mathrm{CF}$ bronchiectasis versus non-CF bronchiectasis.

2. Relative proportion of various bacterial species identified in the respiratory tract in people with $\mathrm{CF}$ bronchiectasis versus non-CF bronchiectasis.

3. Number of bacterial species identified per person with $\mathrm{CF}$ bronchiectasis versus non-CF bronchiectasis.

4. Bacteria identified in people with non-CF bronchiectasis, with a known cause versus unknown cause(s).

5. Proportion of specific bacterial species susceptible to specific antimicrobial agents.

6. Proportion of specific bacterial species resistant to specific antimicrobial agents.

7. Proportion of specific bacterial species with intermediate susceptibility to specific antimicrobial agents.

8. Time-trend of antimicrobial sensitivity patterns in 10-year epochs.

\section{Search methods for identification of studies}

Two authors will independently search the electronic databases PubMed, EMBASE, the Cochrane Library, and LIVIVO. Grey literature will be searched using the search engines, Open Grey and Google Scholar. Figure 1 presents a flowchart of the systematic review. MeSH terms for keywords will be used as follows:

1. Bronchiectasis AND Cystic fibrosis AND non-cystic fibrosis AND (antibiotic OR antimicrobial) AND (susceptibility OR sensitivity OR resistance)

2. Cystic fibrosis AND bronchiectasis AND (antibiotic OR antimicrobial) AND (susceptibility OR sensitivity OR resistance)

3. Non-cystic fibrosis AND bronchiectasis AND (antibiotic OR antimicrobial) AND (susceptibility OR sensitivity OR resistance)

4. Pulmonary exacerbation AND (antibiotic OR antimicrobial) AND (susceptibility OR sensitivity OR resistance)

5. Bronchiectasis AND Cystic fibrosis AND non-cystic fibrosis AND (microbiota OR pathogens OR colonizers OR bacteria OR microbiology)

6. Cystic fibrosis AND bronchiectasis AND (microbiota OR pathogens OR colonizers OR bacteria OR microbiology)

7. Non-cystic fibrosis AND bronchiectasis AND (microbiota OR pathogens OR colonizers OR bacteria OR microbiology)

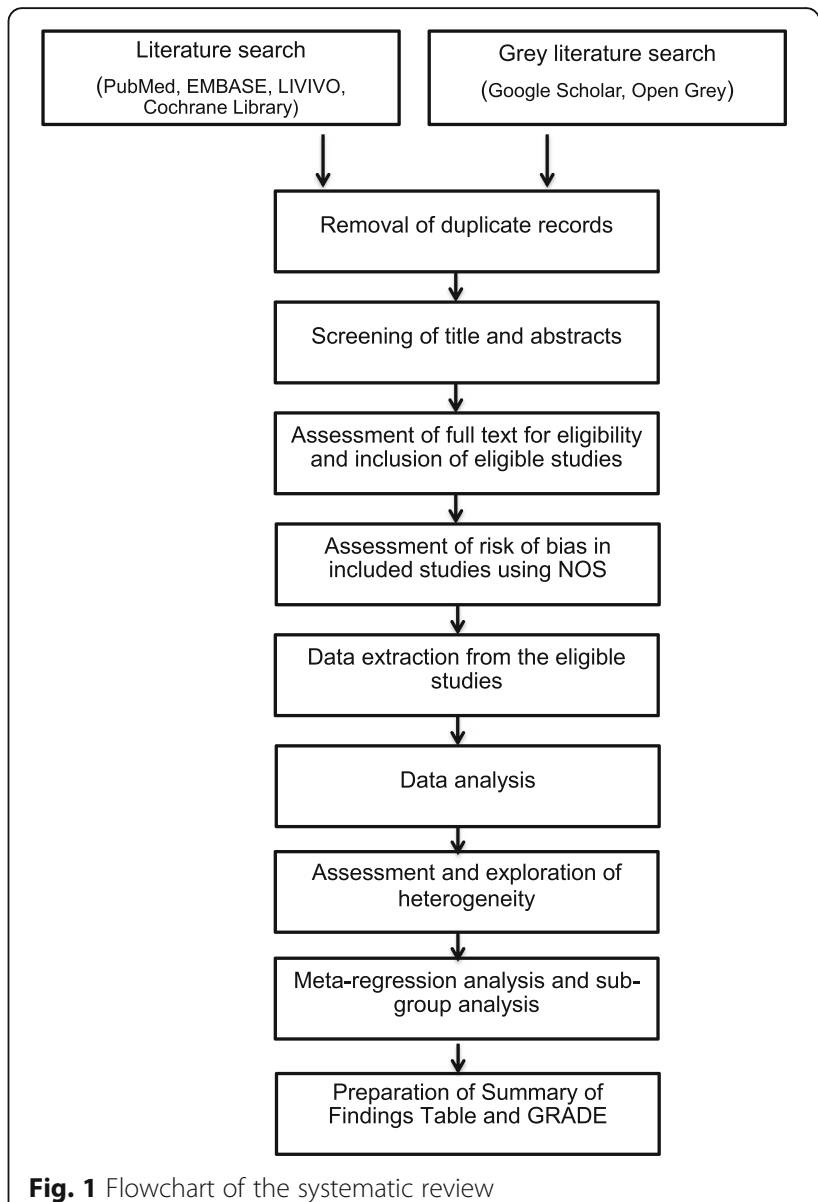

Data collection and analysis

Studies will be screened by examining titles and abstracts. Those identified as potentially relevant will be retrieved and full text examined. Each full-text article will be evaluated for eligibility to be included in the review. There will be no language restriction. Manual search of the reference lists of the included studies will also be performed. We will inform the date last searched for each of the databases. Disagreements/discrepancies will be resolved by discussion and if necessary, arbitration by the third author.

\section{Data extraction and management}

Data will be independently extracted by two reviewers into a special data extraction form.

From each included study, the following information will be extracted: Identification data (author, year), study design, institution, country or countries, time-period of study, whether clinical or microbiological analysis, inclusion criteria of patients (CF and non-CF bronchiectasis), age of patients, clinical state of patients (stable, acute exacerbation, surveillance culture, etc.), underlying clinical condition, duration of illness if known, presence of co- 
morbidity, specimen tested, specimen collection method, whether already on antibiotics, micro-organisms identified, bacteriological method used for identification (culture, molecular methods, etc.), bacterial quantification, biofilm formation, antimicrobial susceptibility profile, method of testing antimicrobial susceptibility, definition of sensitivity, and clinical/bacteriological outcome (Tables 1 and 2).

\section{Assessment of risk of bias in included studies}

Two authors will independently assess the risk of bias in each included study using the Newcastle Ottawa Scale (NOS) [20]. The NOS contains eight items, categorized into three broad perspectives: selection of the study groups, comparability of the groups, and ascertainment of either the exposure or outcome of interest for casecontrol or cohort studies, respectively. For each item, a series of response options is provided. A star system is used to allow a semi-quantitative assessment of study quality. A study can be awarded a maximum of one star for each numbered item within the selection and exposure categories. A maximum of two stars can be given for comparability. High-quality studies will be defined as those having an NOS score $\geq 6$, from a maximum of 9 points [21].

\section{Statistical analysis}

We will present the data with descriptive statistics and provide pooled estimates of outcome parameters, wherever it is feasible to perform meta-analysis. Pooled estimates will be presented with a $95 \%$ confidence interval. The default analysis will be with a random effects model.

\section{Dealing with missing data}

We will contact the corresponding authors of studies where data is/are missing and try to obtain the missing data. If this fails, we will try and impute data where possible. If that is not feasible, we will state as such.

\section{Assessment of heterogeneity}

Heterogeneity in studies will be explored by visual inspection of forest plot as well as using the Higgins and Thompson $I^{2}$ method [22]. The $I^{2}$ heterogeneity will be categorized as follows: $0-50 \%$ low, $50-75 \%$ moderate, and $>75 \%$ considerable heterogeneity. Where $I^{2}$ is greater than $50 \%$, we will try to identify possible explanations using subgroup analysis and meta-regression analysis based on the most important characteristics of the studies.

\section{Assessment of reporting biases}

Wherever possible, we will obtain the original trial protocols for comparison with the published papers to ensure that all outcomes were reported. If it is not possible to obtain the trial protocols, we will scrutinize the "Methods" section of the published paper(s) to ensure full reporting of all measured variables. If negative data were not fully reported, we will contact the primary investigators for these data. We will explore reporting bias using a funnel plot. We will also assess publication bias by looking for evidence of conference presentations not followed by subsequent journal publications.

\section{Sensitivity analysis}

In order to assess whether the results are substantially influenced by the presence of any individual study, we will perform a sensitivity analysis by systematically removing studies with a high risk of bias and recalculating the results.

\section{Subgroup analysis and investigation of heterogeneity}

We will analyze results separately by the following characteristics:

1. Age group of patients: Children $(<18$ years) versus adults ( $\geq 18$ years).

2. Antibiotic therapy: Already on antibiotics versus not on antibiotics.

3. Clinical state: During acute exacerbations versus stable clinical state, i.e., surveillance culture.

4. Type of respiratory specimen: Upper respiratory tract specimen versus lower respiratory tract specimen.

5. Method used for identification of bacterial species: Culture versus molecular methods or other nonculture methods.

\section{Summary of findings table and GRADE}

We will present two summaries of findings' tables; one comparing the bacteria identified in respiratory specimens in those with $\mathrm{CF}$ bronchiectasis versus non-CF bronchiectasis (Table 1), and the other comparing antimicrobial susceptibility patterns of individual bacterial species in CF bronchiectasis versus non-CF bronchiectasis (Table 2).

Two reviewers will independently assess the quality of evidence based on five domains, i.e., design (risk of bias), consistency across studies, directness of the evidence, precision of estimates, and presence of publication bias using the Grading of Recommendations Assessment, Development and Evaluation (GRADE) approach [23]. The body of evidence will then be classified as high, moderate, low, and very low.

\section{Discussion}

To date, there are no locally applicable evidence-based guidelines for antimicrobial treatment of non-CF bronchiectasis patients. In general, treatment is based on 
Table 1 Respiratory pathogen colonization in people with cystic fibrosis bronchiectasis versus non-cystic fibrosis bronchiectasis Data extracted by

Reference

Study design

Clinical/microbiology study

$$
\begin{aligned}
& \text { - Observational study } \\
& \text { - Clinical trial } \\
& \text { - Case series with } \geq 10 \text { cases } \\
& \text { - Case series with } \leq 9 \text { case }
\end{aligned}
$$

Time period

Participants

Study group (CF bronchiectasis)
Author \& year

Citation

Country/countries

Institution/s

- CF bronchiectasis

- Non-CF bronchiectasis

- Mixed

- Other

No.

Age

- Adults $\geq 18$ years

- Children $<18$ years

- Mixed (not possible to separate)

Sex

Respiratory pathogens

No. (\%)

Staphylococcus aureus (MSSA)

Staphylococcus aureus (MRSA)

Pseudomonas aeruginosa

Burkholderia 20 epacian complex

Haemophilus influenzae

Stenotrophomonas maltophilia

Achromobacter xylosoxidans

NTM

Streptococcus

Veillonella

Prevotella

Others

Inclusion/Exclusion criteria

Sample type (sputum/BAL/ELF)

Upper respiratory specimen

- Nasal specimen

- Nasopharyngeal specimen

- Oropharyngeal specimen

- Other

Lower respiratory specimen

- Sputum

- Induced sputum

- BAL

- Lung aspirate

- Other

Antibiotic therapy

- Already on antibiotic(s)

- Not on antibiotic(s)

- Unclear

Method of identifying species 
Table 1 Respiratory pathogen colonization in people with cystic fibrosis bronchiectasis versus non-cystic fibrosis bronchiectasis (Continued)

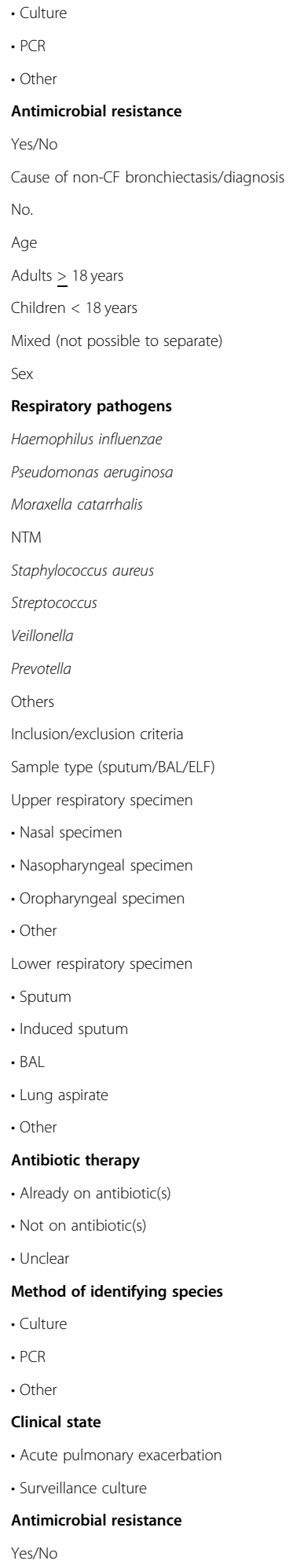


Table 2 Antibiotic susceptibility profile in people with cystic fibrosis versus non cystic fibrosis bronchiectasis

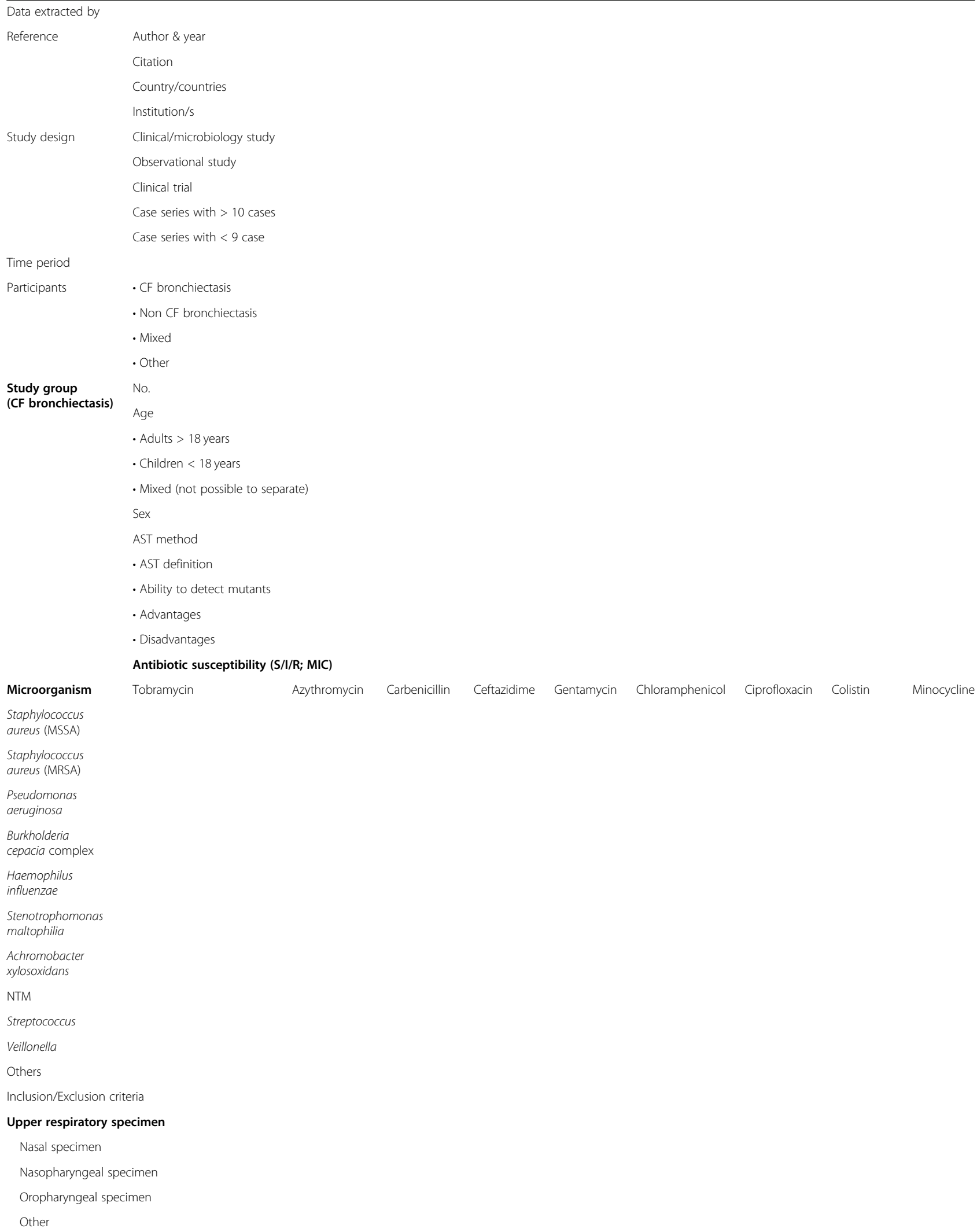

Staphylococcus aureus (MSSA)

Staphylococcus aureus (MRSA)

Pseudomonas aeruginosa

Burkholderia cepacia complex

Haemophilus

influenzae

Stenotrophomonas maltophilia

Achromobacter xylosoxidans

NTM

Streptococcus

Veillonella

Others

Inclusion/Exclusion criteria

Upper respiratory specimen

Nasal specimen

Nasopharyngeal specimen

Oropharyngeal specimen

Other

Lower respiratory specimen 
Table 2 Antibiotic susceptibility profile in people with cystic fibrosis versus non cystic fibrosis bronchiectasis (Continued)

\author{
Sputum \\ Induced sputum \\ BAL \\ Lung aspirate \\ Other \\ Antibiotic therapy \\ Already on antibiotic(s) \\ Not on antibiotic(s) \\ Unclear
}

Method of identifying species

Culture

PCR

Other

Biofilm detection

Antimicrobial resistance (Yes/No)

Remarks

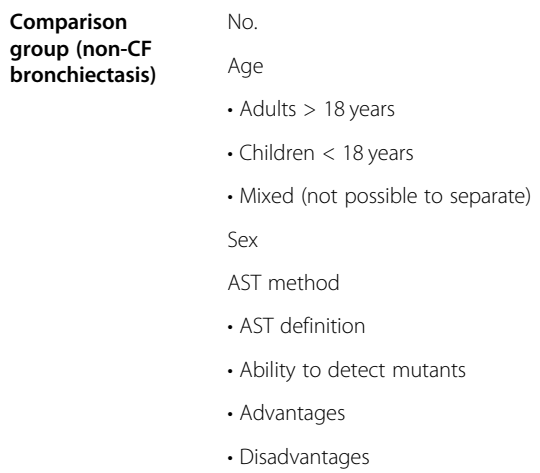

Ciprofloxacin Ceftazidime Cefuroxime Cefotaxime

Flucloxacillin

Tobramycin

Haemophilus

influenzae

Pseudomonas

aeruginosa

Moraxella

catarrhalis

NTM

Staphylococcus

aureus

Streptococcus

Veillonella

Prevotella

Others

Inclusion/Exclusion criteria

Upper respiratory specimen

Nasal specimen

Nasopharyngeal specimen

Oropharyngeal specimen

Other

Lower respiratory specimen

Sputum

Induced sputum 
Table 2 Antibiotic susceptibility profile in people with cystic fibrosis versus non cystic fibrosis bronchiectasis (Continued)

\author{
BAL \\ Lung aspirate \\ Other \\ Antibiotic therapy \\ Already on antibiotic(s) \\ Not on antibiotic(s) \\ Unclear \\ Method of identifying species \\ Culture \\ $\mathrm{PCR}$ \\ Other \\ Biofilm detection \\ Antimicrobial resistance (Yes/No) \\ Remarks
}

extrapolation of evidence in CF bronchiectasis [24, 25]. Hence, comparing the bacterial colonization pattern and antibiotic susceptibility profile in CF bronchiectasis versus non-CF bronchiectasis would aid in improved management of both the conditions. Furthermore, the understanding of the microbiota in both $\mathrm{CF}$ and non-CF populations would aid in more personalized treatment approaches. Understanding the antimicrobial susceptibility patterns against specific organisms can facilitate appropriate rather than empiric therapy and hopefully reduce the burden of antimicrobial resistance created by rampant usage of antibiotics.

\section{Supplementary Information}

The online version contains supplementary material available at https://doi. org/10.1186/s13643-020-01557-6.

Additional file 1. PRISMA-P 2015 Checklist.

\section{Abbreviations}

CF: Cystic fibrosis; AST: Antimicrobial susceptibility; NOS: Newcastle Ottawa Scale; MeSH: Medical Subject Headings; PROSPERO: Prospective Register of Systematic Reviews; MIC: Minimum Inhibitory Concentration;

BAL: Bronchoalveolar lavage; ELF: Epithelial lining fluid; GRADE: Grading of Recommendations Assessment, Development and Evaluation;

PCR: Polymerase chain reaction

\section{Acknowledgements}

None

\section{Authors' contributions}

JLM conceptualized the systematic review and outlined the Objectives, Methods, and Outcomes. SV drafted the protocol. PR provided critical intellectual inputs. The author(s) read and approved the final manuscript.

\section{Funding}

No specific funding has been allocated for the study.

\section{Availability of data and materials}

Not applicable.

Ethics approval and consent to participate Not applicable.
Consent for publication

Not applicable.

\section{Competing interests}

The authors declare that they have no competing interests.

\section{Author details}

${ }^{1}$ Department of Medical Microbiology, Post Graduate Institute of Medical Education and Research, Chandigarh 160012, India. ${ }^{2}$ Department of Pediatrics, Post Graduate Institute of Medical Education and Research, Chandigarh 160012, India.

Received: 3 August 2020 Accepted: 9 December 2020

Published online: 04 January 2021

References

1. Boyle MP, De Boeck K. A new era in the treatment of cystic fibrosis: correction of the underlying CFTR defect. Lancet Respir Med. 2013;1(2):15863.

2. Morrison CB, Markovetz MR, Ehre C. Mucus, mucins, and cystic fibrosis. Pediatr Pulmonol. 2019;54(S3):S84-96.

3. Wine JJ, Hansson GC, König P, Joo NS, Ermund A, Pieper M. Progress in understanding mucus abnormalities in cystic fibrosis airways. I Cyst Fibros. 2018;17(2):S35-9.

4. Hauser AR, Jain M, Bar-Meir M, McColley SA. Clinical significance of microbial infection and adaptation in cystic fibrosis. Clin Microbiol Rev. 2011;24(1):29-70.

5. McShane PJ, Naureckas ET, Tino G, Strek ME. Non-cystic fibrosis bronchiectasis. Am J Respir Crit Care Med. 2013;188(6):647-56.

6. Schäfer J, Griese M, Chandrasekaran R, Chotirmall SH, Hartl D. Pathogenesis, imaging and clinical characteristics of CF and non-CF bronchiectasis. BMC Pulm Med. 2018;18(1):79.

7. Imam JS, Duarte AG. Non-CF bronchiectasis: orphan disease no longer. Respir Med. 2020;166:105940.

8. Cuthbertson L, Walker AW, Oliver AE, Rogers GB, Rivett DW, Hampton TH, et al. Lung function and microbiota diversity in cystic fibrosis. Microbiome. 2020;8:1-3.

9. Richardson H, Dicker AJ, Barclay H, Chalmers JD. The microbiome in bronchiectasis. ERR. 2019;28(153):190048.

10. King P, Holdsworth S, Freezer NJ, Villanueva E, Holmes PW. Microbiologic follow-up study in adult bronchiectasis. Respir Med. 2007;101(8):1633-8.

11. van der Gast CJ, Cuthbertson L, Rogers GB, Pope C, Marsh RL, Redding GJ, et al. Three clinically distinct chronic pediatric airway infections share a common core microbiota. Ann Am Thorac Soc. 2014;11(7):1039-48.

12. Bell SC, Mall MA, Gutierrez H, Macek M, Madge S, Davies JC, et al. The future of cystic fibrosis care: a global perspective. Lancet Respir Med. 2020;8(1):65124.

13. Turnidge J, Paterson DL. Setting and revising antibacterial susceptibility breakpoints. Clin Microbiol Rev. 2007;20(3):391-408. 
14. Molloy L, Nichols K. Infectious diseases pharmacotherapy for children with cystic fibrosis. J Pediatr Heal Care. 2015;29(6):565-78.

15. Riquelme SA, Lung TWF, Prince A. Pulmonary pathogens adapt to immune signaling metabolites in the airway. Front Immunol. 2020;11:385.

16. Kahl BC, Duebbers A, Lubritz G, Haeberle J, Koch HG, Ritzerfeld B, et al. Population dynamics of persistent staphylococcus aureus isolated from the airways of cystic fibrosis patients during a 6-year prospective study. J Clin Microbiol. 2003:41(9):4424-7.

17. Häußler S, Lehmann C, Breselge C, Rohde M, Claßen M, Tümmler B, et al. Fatal outcome of lung transplantation in cystic fibrosis patients due to small-colony variants of the Burkholderia cepacia complex. Eur J Clin Microbiol Infect Dis. 2003;22(4):249-53.

18. Mowat E, Paterson S, Fothergill JL, Wright EA, Ledson MJ, Walshaw MJ, et al. Pseudomonas aeruginosa population diversity and turnover in cystic fibrosis chronic infections. Am J Respir Crit Care Med. 2011:183(12):1674-9.

19. Shamseer L, Moher D, Clarke M, Ghersi D, Liberati A, Petticrew M, et al. Preferred reporting items for systematic review and meta-analysis protocols (PRISMA-P) 2015: elaboration and explanation. BMJ. 2015;349(1):g7647.

20. Wells G, Shea B, O'connell DL, Peterson J, Welch, Losos M, Tugwell P. The Newcastle-Ottawa Scale (NOS) for assessing the quality of nonrandomised studies in meta-analyses. 2018. Available from: http://www.ohri.ca/ programs/clinical_epidemiology/oxford.asp.

21. Stang A. Critical evaluation of the Newcastle-Ottawa scale for the assessment of the quality of nonrandomized studies in meta-analyses. Eur J Epidemiol. 2010;25(9):603-5.

22. Higgins JPT, Savovic J, Page MJ, Elbers RG, JAC S. Chapter 8: Assessing risk of bias in a randomized trial. Cochrane Handbook for Systematic Reviews of Interventions version 6.0 (updated 2019): Cochrane; 2019. Available from: https://training.cochrane.org/handbook/current/chapter-08.

23. Balshem $H$, Helfand M, Schünemann CJ, Oxman AD, Kunz R, Brozek J, et al. GRADE guidelines: 3. Rating the quality of evidence. J Clin Epidemiol. 2011; 64:401-6.

24. Aliberti S, Masefield S, Polverino E, De Soyza A, Loebinger MR, Menendez R, et al. Research priorities in bronchiectasis: a consensus statement from the EMBARC Clinical Research Collaboration. Eur Respir J. 2016:48(3):632-47.

25. Pasteur MC, Bilton D, Hill AT. British Thoracic Society guideline for non-CF bronchiectasis. Thorax. 2010;65:11-58.

\section{Publisher's Note}

Springer Nature remains neutral with regard to jurisdictional claims in published maps and institutional affiliations.

Ready to submit your research? Choose BMC and benefit from:

- fast, convenient online submission

- thorough peer review by experienced researchers in your field

- rapid publication on acceptance

- support for research data, including large and complex data types

- gold Open Access which fosters wider collaboration and increased citations

- maximum visibility for your research: over $100 \mathrm{M}$ website views per year

At $\mathrm{BMC}$, research is always in progress.

Learn more biomedcentral.com/submissions 